ARTIGO ORIGINAL

\title{
Perfil dietético e risco cardiovascular em adolescentes de uma escola privada de São Paulo
}

\author{
Dietary patterns and cardiovascular risk in adolescents from a private school in São Paulo
}

\author{
Marina Brosso Pioltine ${ }^{1}$, Ana Carolina Almada Colucci Paternez ${ }^{2}$, \\ Juliana Masami Morimoto ${ }^{3}$
}

\begin{abstract}
RESUMO
Introdução: As doenças cardiovasculares são consideradas um importante problema de saúde pública e torna-se importante estudar estratégias que permitam o seu controle desde a adolescência.

Objetivo: Avaliar o consumo alimentar e identificar os fatores dietéticos protetores e de risco para doenças cardiovasculares em adolescentes de uma escola privada de São Paulo, Brasil.

Materiais e Métodos: Estudo realizado com 48 adolescentes no período de agosto de 2011 a junho de 2012 . O estado nutricional foi avaliado pelas curvas de crescimento da Organização Mundial da Saúde. O consumo alimentar foi analisado pelo registro alimentar de três dias, segundo as Dietary Reference Intakes. Considerou-se como fator de risco cardiovascular o sedentarismo, história familiar para doença cardiovascular, tabagismo, consumo alcoólico e tempo gasto em atividades sedentárias, como fazer uso de televisão, computador e videogames.

Resultados: Verificou-se a elevada prevalência de obesidade nos adolescentes de ambos os gêneros, sendo $16,67 \%$ nas meninas e 5,56\% nos meninos. Quando associado ao sobrepeso, os valores foram maiores nas meninas (43,34\%), quando comparado aos meninos (40,29\%). Constatou-se elevada ingestão de gorduras totais $(33,33 \%)$, gorduras saturadas $(37,50 \%)$, gorduras poli-insaturadas $(39,58 \%)$ e sódio $(37,50 \%)$. Verificou-se o baixo consumo de gorduras monoinsaturadas $(89,58 \%)$ e fibras (100\%). Quanto aos fatores de risco associados, verificou-se elevada prevalência de sedentarismo $(81,25 \%)$, histórico familiar de doenças cardiovasculares $(75 \%)$ e elevado tempo gasto em atividades sedentárias $(87,50 \%)$.

Conclusão: Houve elevada inadequação do consumo alimentar e alta prevalência de fatores de risco cardiovascular na população estudada, sendo necessária a intervenção com foco em hábitos alimentares e estilo de vida saudáveis.
\end{abstract}

Palavras-chave: consumo alimentar; adolescentes; doenças cardiovasculares.

\begin{abstract}
Introduction: Cardiovascular diseases are considered a public health problem and it is important to study strategies for its control since adolescence.

Objective: To evaluate the dietary intake and to identify risk factors for cardiovascular diseases in adolescents from a private school in Sao Paulo, Brazil.

Materials and Methods: The study was conducted with 48 adolescents from August 2011 to June 2012. The nutritional status was assessed by the Growth Curves of the World Health Organization. Food intake was analyzed by a three-day food record, according to the Dietary Reference Intakes. It was considered as a cardiovascular risk factor: physical inactivity, family history of cardiovascular disease, smoking, alcohol consumption and time spent in sedentary activities such as television, computer and videogames.

Results: There was a high prevalence of obesity in adolescents of both genders (16.67\% females and 5.56\% males). When associated with overweight, the levels were higher in girls (43.34\%) compared to boys (40.29\%). The study found an increased intake of total fat (33.33\%), saturated fat (37.50\%), polyunsaturated fat (39.58\%) and sodium (37.50\%). There was a low intake of monounsaturated fat (89.58\%) and fibers (100\%). Regarding risk factors, there was a high prevalence of physical inactivity (81.25\%), family history of cardiovascular disease (75\%) and high time spent in sedentary activities (87.50\%).

Conclusions: There was a high inadequacy in the dietary habits of adolescents and it was found a high prevalence of cardiovascular risk factors, being necessary an intervention focused on diet and healthy lifestyles.
\end{abstract}

Keywords: food consumption; adolescents; cardiovascular diseases.

\footnotetext{
${ }^{1}$ Nutricionista. Mestranda em Ciências, Endocrinologia, pela Faculdade de Medicina da Universidade de São Paulo (USP).

${ }^{2}$ Nutricionista. Doutora em Saúde Pública pela Faculdade de Saúde Pública da USP. Docente do Curso de Nutrição, do Centro de Ciências Biológicas e da Saúde, Universidade Presbiteriana Mackenzie, São Paulo, SP, Brasil.

${ }^{3}$ Nutricionista. Doutora em Ciências, Nutrição em Saúde Pública, pela Faculdade de Saúde Pública da USP. Docente do Curso de Nutrição, do Centro de Ciências Biológicas e da Saúde, Universidade Presbiteriana Mackenzie, São Paulo, SP, Brasil.
} 


\section{INTRODUÇÃO}

As doenças cardiovasculares (DCV) constituem a principal causa de mortalidade no mundo e representam cerca de $30 \%$ das causas de óbito ${ }^{1,2}$. Trata-se de um importante problema de saúde pública, tendo em vista que altas prevalências são encontradas em países com economias emergentes e atingem todos os grupos socioeconômicos, independente da faixa etária, gênero ou etnia ${ }^{3}$.

Embora as DCV apresentem manifestação clínica na idade adulta, é evidente que os fatores de risco surgem cada vez mais cedo e se estendem a idades mais avançadas $^{4}$. Ainda que seja possível observar o progresso no tratamento das DCV, as medidas preventivas são a forma mais eficaz de diminuir a elevada prevalência global destas enfermidades ${ }^{5}$.

Os fatores de risco para DCV na adolescência são o excesso de peso, hipertensão arterial, dislipidemias, diabetes mellitus, sedentarismo e história familiar positiva para doença cardiovascular. O tabagismo e o consumo excessivo de álcool também são importantes fatores de risco na adolescência, pois fazem parte de comportamentos que podem ter início nesta etapa da vida $^{6}$. Sabe-se que a presença de fatores de risco indica uma probabilidade maior para o surgimento futuro de uma enfermidade, e quando há a associação entre mais de um fator de risco, a probabilidade de ocorrência de eventos clínicos torna-se exponencialmente maior, fazendo-se necessária a intervenção precoce ${ }^{7}$.

O padrão dietético rico em frutas, hortaliças, fibras, minerais e laticínios com baixo teor de gordura, tem importante impacto na redução do risco cardiovascular. O elevado grau de adesão a esse tipo de dieta reduz a probabilidade de desenvolver DCV, devido às quantidades significativas de potássio, magnésio e cálcio, além de ser um padrão alimentar que potencializa o efeito de orientações nutricionais para emagrecimento, reduzindo também a presença de biomarcadores de risco cardiovascular ${ }^{3}$.

Os hábitos alimentares saudáveis promovem a prevenção das DCV por meio de mecanismos fisiológicos, como a redução da glicemia, redução da absorção enteral do colesterol, inibição da oxidação da lipoproteína LDL e controle dos níveis pressóricos do sangue $^{1}$. O consumo de fibras dietéticas e de gorduras monoinsaturadas, como o ômega 9, e gorduras poliinsaturadas, como o ômega 3 e ômega 6 , em quantidades adequadas, também contribui para a saúde cardiovascular ${ }^{8}$. Por outro lado, a ingestão excessiva de sódio, álcool, açúcares, gorduras totais, gorduras saturadas, gorduras trans e alimentos de elevada densidade energética tem sido correlacionada com o excesso de peso e elevado risco cardiovascular ${ }^{9}$.

Verificar a prevalência de fatores de risco cardiovascular em idades precoces torna-se prioridade, uma vez que contribui para a elaboração de estratégias de promoção à saúde, com ações para a manutenção e adoção de hábitos saudáveis que, incorporados na infância e adolescência, podem ser mantidos até a idade adulta ${ }^{10}$. Destaca-se também que a escola é um local propício para a adoção de medidas educativas e preventivas no que diz respeito aos hábitos alimentares, uma vez que se trata de um ambiente de aprendizado e com foco em indivíduos em fase de formação de novos hábitos ${ }^{10}$

Assim, o objetivo do estudo foi avaliar o consumo alimentar e a presença de fatores de risco cardiovascular em adolescentes de uma escola privada da cidade de São Paulo, SP, Brasil.

\section{MATERIAIS E MÉTODOS}

Trata-se de um estudo de prevalência, com abordagem semi-quantitativa e quantitativa da alimentação de adolescentes. O estudo foi realizado no período de agosto de 2011 a junho de 2012.

O estudo foi conduzido com adolescentes de 15 e 16 anos de idade de ambos os gêneros, matriculados no primeiro e segundo ano do ensino médio de uma instituição de ensino privada, localizada na região central da cidade de São Paulo (São Paulo, Brasil). Trata-se de uma amostra de conveniência onde todos os alunos matriculados nestes anos foram convidados a participarem do estudo.

Antes do início da pesquisa, foi enviado à escola uma Carta de Informação à Instituição, com a apresentação da pesquisa. Após aprovação por parte da Instituição de Ensino, encaminhou-se o Termo de Consentimento Livre e Esclarecido aos pais dos alunos, solicitando 
suas autorizações para a participação no estudo

Os adolescentes foram classificados em baixo peso, eutrofia, sobrepeso ou obesidade a partir do IMC por idade (IMC/I), conforme proposto pelas curvas de crescimento da Organização Mundial da Saúde ${ }^{11}$. Foram caracterizados com sobrepeso os adolescentes que apresentaram IMC acima do percentil 85 e com obesidade aqueles com IMC acima do percentil 95.

O consumo alimentar foi avaliado através do preenchimento do registro alimentar de três dias, por parte dos adolescentes, em dias alternados, sendo dois dias não consecutivos durante a semana, e um dia ao final de semana. Foi realizada uma orientação detalhada quanto à forma correta de preenchimento do registro alimentar, que foi preenchido em horários não acadêmicos e devolvidos em um período máximo de duas semanas. Foi realizada uma análise crítica dos alimentos descritos, a fim de identificar possíveis preparações de receitas caseiras, para que fosse realizada uma padronização nas medidas, em gramas, do consumo de cada ingrediente. As medidas caseiras presentes no registro alimentar de três dias foram transformadas em gramas, utilizando-se a tabela elaborada por Pinheiro et al. ${ }^{12}$.

Analisou-se a média das refeições realizadas por dia e a frequência de realização de cada refeição. Para tanto, considerou-se como desjejum as refeições realizadas entre o período das $05 \mathrm{~h} 30$ às $09 \mathrm{~h} 00$, colação entre o período das $09 \mathrm{~h} 00$ às $11 \mathrm{~h} 30$, almoço entre $11 \mathrm{~h} 30$ e 14h30, lanche da tarde das $14 \mathrm{~h} 30$ às 18h00, jantar das $18 \mathrm{~h} 00$ às $20 \mathrm{~h} 30$ e ceia das $20 \mathrm{~h} 30$ às $22 \mathrm{~h} 30$. Nos momentos em que a refeição não foi informada, segundo o critério de horários adotado, considerou-se a sua omissão.

Foi realizada uma análise semi-quantitativa da dieta, onde os alimentos descritos nos registros alimentares foram quantificados em porções segundo o Guia Alimentar para a População Brasileira ${ }^{9}$ para a análise do consumo dos grupos alimentares. Além disso, analisouse quais foram os alimentos de maior frequência no consumo dos adolescentes. Quando houve a presença de alimentos preparados em casa ou restaurantes acrescentou-se $8 \mathrm{ml}$ de óleo (1 colher de sopa por dia) ao cálculo da dieta como óleo utilizado para a cocção.
Não se acrescentou o óleo para cocção quando o adolescente consumiu apenas produtos industrializados no decorrer do dia.

Calculou-se o valor energético total (VET), carboidratos, proteínas, ácidos graxos totais, monoinsaturados, poli-insaturados, saturados, colesterol, fibras e sódio utilizando-se o Software Avanutriß versão 4.0. Para a análise da adequação dos nutrientes foram utilizadas as recomendações do Institute of Medicine ${ }^{13}$, denominadas DRIs (Dietary Reference Intakes) para os carboidratos, proteínas, lipídios totais, fibras dietéticas e sódio, sendo que este último foi analisado segundo valores de Al (Adequate Intakes) e UL (Tolerable Upper Intake Levels). Utilizouse recomendações da Organização Mundial da Saúde ${ }^{14}$ para as frações de lipídios dietéticos (gorduras saturadas, monoinsaturadas, poli-insaturadas e colesterol).

Quanto aos fatores de risco cardiovascular associados, estes foram avaliados por meio de um questionário, elaborado pelas pesquisadoras, com perguntas sobre o histórico de DCVs na família (pais, tios e avós), prática de atividades físicas (modalidade, frequência semanal e duração em minutos), bem como o tempo gasto em atividades sedentárias, como fazer uso de televisão, computador e/ou videogames. Foi também investigado 0 hábito de fumar, sendo 0 adolescente classificado como fumante, não-fumante ou ex-fumante segundo informação referida e, ainda, avaliado o consumo de bebidas alcoólicas. Quanto ao consumo alcoólico avaliou-se o risco de ingestão semanal, independente do tipo de bebida e quantidade ingerida.

A prática de atividades físicas foi avaliada levando-se em consideração a realização de educação física na escola e seguiu a recomendação da Organização Mundial da Saúde ${ }^{15}$ para adolescentes, que equivale a, no mínimo, 60 minutos de atividade moderada por dia. O tempo gasto em atividades sedentárias (televisão, computador e/ou videogames) foi analisado de acordo com a recomendação da American Academy of Pediatrics ${ }^{16}$, sendo classificado como adequado o hábito de uso por até duas horas ao dia.

Todos os procedimentos estatísticos foram realizados 


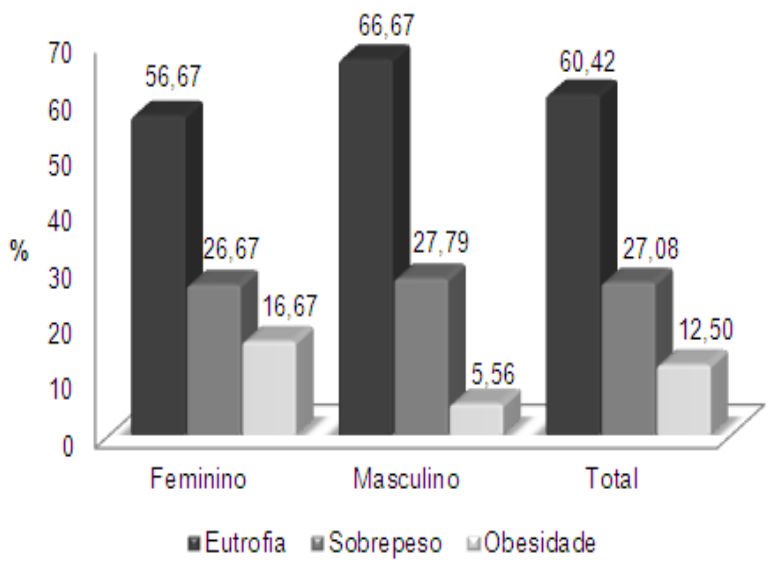

FIGURA 1 - Classificação do estado nutricional de adolescentes de 15 e 16 anos de idade, segundo o índice de massa corporal.

com o Software SPSS® (Statistical Package for Social Science Inc., Chicago, Illinois, USA), versão 20.0, sendo que os resultados qualitativos foram descritos por meio de frequências absolutas e relativas e os quantitativos por média e desvio padrão. O presente estudo foi aprovado pelo Comitê de Ética em Pesquisa da Universidade Presbiteriana Mackenzie sob Processo CEP/UPM no 1383/08/2011 e CAAE no 0078.0.272.00011.

\section{RESULTADOS}

Participaram deste estudo 48 adolescentes, sendo 30 meninas, com média de idade de 15,6 anos $( \pm 0,4)$ e 18 meninos com média de idade de 15,7 anos $( \pm 0,4)$. A figura 1 demonstra as prevalências de eutrofia, sobrepeso e obesidade nos adolescentes de uma escola privada da cidade de São Paulo.

Em relação à alimentação, verificou-se que a média de refeições realizadas por dia pelos adolescentes foi de $4,8( \pm 1,1)$. Verificou-se maior omissão do desjejum por parte dos meninos $(11,11 \%)$ quando comparado às meninas $(7,78 \%)$. Os dados de consumo alimentar dos adolescentes estão descritos na tabela 1, na qual é possível observar o consumo de diferentes grupos alimentares.

Verifica-se o baixo consumo de cereais, tubérculos e raízes em ambos os gêneros (recomendação: 6 porções ao dia), bem como o elevado consumo de açúcares e doces (recomendação: 1 porção ao dia) na população

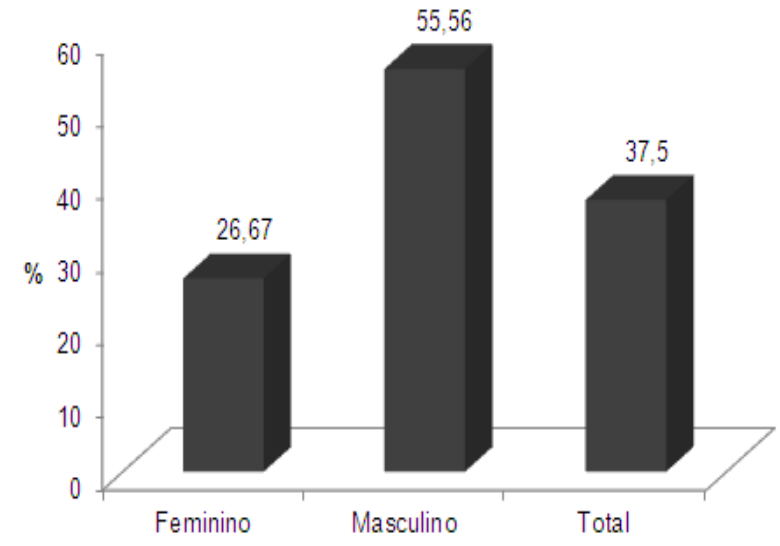

FIGURA 2 - Frequência (\%) de adolescentes que consomem sódio em quantidades acima das recomendadas, segundo gênero.

estudada, principalmente no gênero masculino. O consumo de leguminosas pode ser considerado aceitável, uma vez que seus valores médios estão próximos ao recomendado (1 porção ao dia). Observouse 0 consumo excessivo de carnes e ovos (recomendação: 1 porção ao dia), bem como de óleos e gorduras (recomendação 1 porção ao dia), em detrimento do consumo de frutas (recomendação: 3 porções ao dia), legumes e verduras (recomendação: 3 porções ao dia) e laticínios (recomendação: 3 porções ao dia), nos dois gêneros.

O alimento mais consumido do grupo de cereais, tubérculos e raízes foi 0 arroz branco (5\% pelas meninas e $7,18 \%$ pelos meninos). A leguminosa mais consumida foi o feijão $(2,20 \%$ pelas meninas e $4,38 \%$ pelos meninos). Quanto ao grupo das carnes e ovos, verificou-se que o alimento mais consumido foi a carne bovina (5,51\% pelas meninas e $4,50 \%$ pelos meninos). $\mathrm{O}$ produto lácteo mais consumido pelas meninas foi 0 queijo $(3,64 \%)$, enquanto que nos meninos foi o leite integral $(4,50 \%)$. Entre os legumes e hortaliças mais consumidos pelas meninas, foi referido o tomate $(2,02 \%)$, enquanto que nos meninos foi o alface $(2,55 \%)$. A fruta mais consumida pelas meninas foi a laranja $(1,02 \%)$ e, nos meninos, este dado não foi verificado devido ao baixo consumo deste grupo alimentar (citação menor que 1\%).

$O$ doce mais consumido pelos adolescentes foi 0 achocolatado em pó $(4,92 \%$ nas meninas e 3,41\% pelos meninos). Quanto ao grupo de óleos e gorduras o 
TABELA 1 - Consumo de porções alimentares, em média e desvio padrão, segundo grupo alimentar e gênero.

\begin{tabular}{lcccc}
\hline Grupo alimentar & Feminino & Masculino & Total & Recomendação \\
\hline Cereais, tubérculos e raízes & $4,04 \pm 2,34$ & $5,11 \pm 4,32$ & $4,45 \pm 3,27$ & 6 \\
Leguminosas & $0,91 \pm 0,54$ & $1,19 \pm 0,72$ & $1,07 \pm 0,66$ & 1 \\
Carnes e ovos & $2,03 \pm 1,22$ & $2,48 \pm 1,82$ & $2,20 \pm 1,48$ & 1 \\
Produtos lácteos & $1,25 \pm 0,75$ & $1,84 \pm 1,20$ & $1,50 \pm 1,00$ & 3 \\
Frutas, legumes e verduras & $0,93 \pm 0,91$ & $0,96 \pm 0,78$ & $0,94 \pm 0,85$ & $6^{*}$ \\
Açúcares e doces & $2,51 \pm 1,64$ & $3,30 \pm 3,55$ & $2,79 \pm 2,48$ & 1 \\
Óleos e gorduras & $1,26 \pm 0,48$ & $1,47 \pm 0,91$ & $1,34 \pm 0,68$ & 1 \\
\hline
\end{tabular}

*a recomendação diária é de três porções de frutas e três porções de legumes e hortaliças, totalizando seis porções diárias.

produto mais consumido foi o óleo utilizado na cocção dos alimentos, sendo que este não foi relatado pelos participantes, e sim, acrescentado ao cálculo da dieta pela pesquisadora, quando houve a presença de alimentos preparados em casa ou restaurantes (não se acrescentou o óleo para cocção quando o adolescente consumiu apenas produtos industrializados no decorrer do dia).

Os dados do consumo de macronutrientes, frações de lipídios, fibras e sódio, segundo o gênero, podem ser observados na tabela ${ }^{2}$. Já as adequações do consumo alimentar dos adolescentes de São Paulo estão descritas na tabela 3 . A figura 2 demonstra a porcentagem de adolescentes que consome sódio acima dos valores máximos recomendados (UL).

No que diz respeito aos fatores de risco cardiovascular associados, os dados podem ser analisados por meio da tabela 4. Observa-se elevada prevalência de sedentarismo e presença de história familiar positiva para DCV em ambos os gêneros, principalmente nas meninas.

Verifica-se o elevado tempo gasto em atividades sedentárias pelos adolescentes de ambos os gêneros (90\% das meninas e $83,33 \%$ dos meninos). O tempo médio gasto na televisão, computador e videogame, verificado nos meninos, foi de 4,31 ( $\pm 1,82)$ horas por dia, e nas meninas foi de $4,68( \pm 1,93)$ horas por dia.

\section{DISCUSSÃO}

A prevalência de sobrepeso encontrada neste estudo $(26,67 \%$ nas meninas e $27,79 \%$ nos meninos), bem como a obesidade $(16,67 \%$ nas meninas e $5,56 \%$ nos meninos) foi elevada, como demonstrou a Pesquisa de Orçamentos Familiares (POF) 2008-200917, na qual identificou-se, em adolescentes de 10 a 19 anos de idade da região sudeste e zona urbana do país, a prevalência de sobrepeso em $20,9 \%$ das meninas e em $24,5 \%$ dos meninos, bem como $4,8 \%$ de obesidade em meninas e $7,6 \%$ em meninos.

Estes achados corroboram a elevada prevalência de sobrepeso e obesidade devido à transição nutricional que ocorre no país, em consequência das modificações no consumo dietético e nos padrões de gasto energético $^{18}$, o que confirma que a obesidade é um importante problema de saúde pública entre adolescentes de países em desenvolvimento, tendo como algumas de suas consequências o maior risco no desenvolvimento de hipertensão arterial, aterosclerose, alterações no perfil lipídico e glicídico, alterações hormonais, complicações nas articulações, distúrbios psicológicos, carências nutricionais e predisposição para o surgimento de alguns tipos de câncer².

Ao analisar-se o consumo alimentar dos adolescentes de São Paulo, observa-se o consumo excessivo de gorduras totais, gorduras saturadas e colesterol, principalmente no gênero masculino, além do consumo insuficiente de gorduras monoinsaturadas e fibras em ambos os gêneros. Alerta-se, ainda, para a elevada prevalência do consumo insuficiente de carboidratos por parte dos meninos, o que aumenta o consumo de calorias provenientes de gorduras. O padrão reduzido de consumo de carboidratos aqui observado foi também identificado na população brasileira, de acordo com dados mais recentes da POF 2008-200919 que, com base em dados sobre a disponibilidade domiciliar de alimentos, demonstram contribuição percentual de $59,2 \%$ dos carboidratos ao total energético da dieta. Ainda, em avaliação da tendência secular do padrão 
TABELA 2 - Consumo alimentar de adolescentes de 15 e 16 anos de idade, em média e desvio padrão, segundo gênero.

\begin{tabular}{lccc}
\hline Nutrientes & Feminino & Masculino & Total \\
\hline Carboidratos (\%) & $55,00 \pm 5,56$ & $49,80 \pm 10,70$ & $53,05 \pm 8,19$ \\
Proteínas (\%) & $14,65 \pm 3,02$ & $18,12 \pm 6,49$ & $15,95 \pm 4,87$ \\
Gorduras totais (\%) & $30,44 \pm 5,56$ & $32,08 \pm 7,61$ & $31,05 \pm 6,38$ \\
Gorduras saturadas (\%) & $9,14 \pm 2,99$ & $10,73 \pm 3,62$ & $9,74 \pm 3,30$ \\
Gorduras monoinsaturadas (\%) & $5,44 \pm 1,59$ & $7,87 \pm 2,86$ & $6,35 \pm 2,43$ \\
Gorduras poli-insaturadas (\%) & $4,33 \pm 2,32$ & $3,78 \pm 2,21$ & $4,12 \pm 2,27$ \\
Colesterol (mg/dia) & $184,34 \pm 47,31$ & $259,09 \pm 104,90$ & $212,37 \pm 81,85$ \\
Fibras (g/dia) & $11,93 \pm 3,81$ & $14,28 \pm 6,71$ & $12,81 \pm 5,15$ \\
Sódio (mg/dia) & $1978,63 \pm 518,92$ & $2344,63 \pm 864,94$ & $2115,88 \pm 684,7$ \\
\hline
\end{tabular}

Dados apresentados em média \pm desvio padrão.

alimentar da população brasileira nos últimos 30 anos, evidencia-se diminuição na participação relativa de carboidratos totais e complexos - estes associados ao maior teor de fibras e à maior densidade nutritiva - e constata-se a ocorrência de deslocamento da disponibilidade de carboidratos por gorduras ${ }^{9}$.

O presente estudo também aponta que as meninas possuem maior ingestão de carboidratos e gorduras poli-insaturadas, quando comparadas aos meninos, que por sua vez consomem mais proteínas, colesterol, fibras, sódio, gorduras totais, saturadas e monoinsaturadas. A inadequação alimentar encontrada no presente estudo se assemelha ao encontrado na POF 2008-2009 ${ }^{19}$, onde verificou-se a inadequação do consumo de gorduras saturadas em $90 \%$ das meninas de 14 a 18 anos de idade e de $80 \%$ nos meninos da mesma faixa etária. Por meio deste estudo nacional identificou-se que o consumo de fibras foi inadequado em $86 \%$ das meninas e $77 \%$ dos meninos. Também observou-se o consumo de $2914,9 \mathrm{mg}$ de sódio por dia pelas meninas e $3744,3 \mathrm{mg}$ deste nutriente por dia, em meninos residentes da zona urbana ${ }^{19}$.

A omissão do desjejum encontrada neste estudo corrobora com a inadequada ingestão de nutrientes. No café da manhã é possível consumir alimentos ricos em nutrientes que não são obtidos apenas pelas principais refeições, como as frutas, ricas em fibras, vitaminas e minerais, e produtos lácteos, fontes de cálcio ${ }^{20}$. Observa-se que os adolescentes avaliados no presente estudo apresentaram consumo de alimentos como o arroz branco, feijão, carne bovina, produtos lácteos, legumes, hortaliças e doces semelhante ao observado com indivíduos de 10 a 19 anos de todo o país, não levando-se em consideração a renda familiar, localização geográfica e gênero ${ }^{19}$.

O presente estudo apresenta algumas limitações que merecem discussão: foram analisados adolescentes matriculados em escola privada, isto pode representar uma parcela diferenciada da população em geral, pelo acesso à informação e condição financeira, o que pode repercutir sobre seus hábitos alimentares e na prática de atividade física. Outro ponto a destacar diz respeito à investigação do consumo alimentar, pois o registro alimentar de três dias utilizado, ainda que mais preciso quando comparado a outros inquéritos alimentares, é um método que pode apresentar erros, uma vez que não é quantificado o consumo exato de alimentos, tratando-se apenas de uma estimativa, necessitando análise com cautela.

Com relação à atividade física, observou-se que o tempo gasto em atividades sedentárias (televisão, videogame e computador) foi em média de $4,54( \pm 1,88)$ horas por dia, valor este acima do recomendado pela American Academy of Pediatrics ${ }^{16}$, que é de até 2 horas por dia. É importante ressaltar que a prática adequada de atividade física na adolescência traz vários benefícios para a saúde física e mental, seja por meio de uma influência direta sobre a morbidade na própria adolescência ou por uma influência mediada pelo nível de atividade física na idade adulta, apresentando uma relação inversa com risco de doenças crônicas e efeito positivo na qualidade de vida e em outras variáveis 
TABELA 3 - Adequação da ingestão de macronutrientes, colesterol e fibras dietéticas, em porcentagem, segundo gênero.

\begin{tabular}{lccccccccc}
\hline \multirow{2}{*}{ Nutrientes } & \multicolumn{3}{c}{ Feminino } & \multicolumn{3}{c}{ Masculino } & \multicolumn{3}{c}{ Total } \\
\cline { 2 - 9 } & Abaixo & Adequado & Acima & Abaixo & Adequado & Acima & Abaixo & Adequado & Acima \\
\hline Carboidratos & - & 93,33 & 6,67 & 33,33 & 55,56 & 11,11 & 12,5 & 79,17 & 8,33 \\
Proteínas & - & 100 & - & 11,11 & 77,78 & 11,11 & 4,17 & 91,67 & 4,17 \\
Gorduras totais & 6,67 & 70 & 23,33 & 11,11 & 38,89 & 50 & 8,33 & 58,33 & 33,33 \\
AGS $^{*}$ & - & 73,33 & 26,67 & - & 44,44 & 55,56 & - & 62,5 & 37,5 \\
AGM $^{* *}$ & 100 & - & - & 72,22 & 27,78 & - & 89,58 & 10,42 & - \\
AGP $^{* * *}$ & - & 96,67 & 3,33 & - & - & 100 & - & 60,42 & 39,58 \\
Colesterol & - & 96,67 & 3,33 & - & 66,67 & 33,33 & - & 85,42 & 14,58 \\
Fibras & 100 & - & - & 100 & - & - & 100 & - & - \\
\hline${ }^{* a ́ c i d o s ~ g r a x o s ~ s a t u r a d o s ; ~}{ }^{* * a ́ c i d o s ~ g r a x o s ~ m o n o i n s a t u r a d o s ; ~}{ }^{* * * a ́ c i d o s ~ g r a x o s ~ p o l i-i n s a t u r a d o s . ~}$ & & & & & &
\end{tabular}

psicológicas $^{16}$. Também torna-se importante destacar que os adolescentes constituem um grupo vulnerável Desde o ponto de vista nutricional, considerando seu perfil dietético, estilo de vida e sua suscebilidade às influências ambientais ${ }^{21}$.

Por meio deste estudo foi possível concluir que as prevalências de sobrepeso e obesidade são consideradas elevadas nos adolescentes da cidade de São Paulo. Houve elevado consumo de sódio, colesterol, gorduras totais e gorduras saturadas nos adolescentes de ambos os gêneros, enquanto que a ingestão de fibras, gorduras monoinsaturadas e poliinsaturadas foi considerada baixa. Em relação aos fatores de risco cardiovascular associados verificou-se elevada prevalência de sedentarismo, histórico familiar positivo para DCV e tempo em atividades sedentárias. Assim, diante do exposto evidencia-se a importância das medidas de controle e prevenção de fatores de risco, as quais devem ser tomadas precocemente em todos os níveis de atenção à saúde e de forma direcionada às necessidades específicas dos

TABELA 4 - Presença de comportamentos e fatores de risco cardiovascular, em porcentagem, segundo gênero.

\begin{tabular}{lccc}
\hline Fatores de risco & $\begin{array}{c}\text { Feminino } \\
(\%)\end{array}$ & $\begin{array}{c}\text { Masculino } \\
(\%)\end{array}$ & $\begin{array}{c}\text { Total } \\
(\%)\end{array}$ \\
\hline Sedentarismo & 86,70 & 72,22 & 81,25 \\
Tabagismo & - & - & - \\
$\begin{array}{l}\text { Consumo alcoólico } \\
\text { semanal }\end{array}$ & 6,67 & 5,56 & 6,25 \\
Histórico familiar & 76,67 & 72,22 & 75,00 \\
DCV & 90 & 83,33 & 87,50 \\
Tempo de tela* & 90
\end{tabular}

*levando-se em consideração a soma do tempo gasto na televisão, computador e videogame maior que $2 \mathrm{~h}$ por dia. adolescentes, a fim de contribuir para sua melhor qualidade de vida e evitar o surgimento futuro de enfermidades cardiovasculares.

\section{REFERÊNCIAS}

1. Rodrigues AN, Perez AJ, Pires JGP, Carletti L, Araújo MTM, Moyses MR, Bissoli NS, Abreu GR. Cardiovascular risk factors, their associations and presence of metabolic syndrome in adolescents. J Pediatr. 2009;85(1):55-60.

2. World Health Organization. Diet, nutrition and prevention of chronic diseases. Geneva: WHO; 2003. (Technical Report Series, 916)

3. Sociedade Brasileira de Cardiologia. Sociedade Brasileira de Hipertensão. Sociedade Brasileira de Nefrologia. VI Diretrizes Brasileiras de Hipertensão Arterial. Arq Bras Cardiol. 2010;95(1):1-51.

4. Buff CG, Ramos E, Souza FIS, Sarni ROS. Frequência de síndrome metabólica em crianças e adolescentes com sobrepeso e obesidade. Rev Paul Pediatr. 2007;25(3): 221-6.

5. Ogden CL, Carroll MD, Flegal KM. High body mass index for age among US children and adolescents, 2003-2006. JAMA. 2008; 299(20):2401-5.

6. Yoshinaga M, Hatake S, Tachikawa $T$, Shinomiya M, Miyazaki A, Takahashi $H$. Impact of lifestyles of adolescents and their parents on cardiovascular risk factors in adolescents. J Atheroscler Thromb. 2011;18(1): 1-10.

7. Yoshinaga M, Takahashi H, Shinomiya M, Miyazaki A, Kuribayashi N, Ichida FF. Impact of having one cardiovascular risk factor on other cardiovascular risk factor level in adolescents. J Atheroscler Thromb. 2010; 17(1):167-75

8. Fernández LC, Serrab JD, Álvarez JRM, Alberich RS, Jiménez FP. Grasas de la dieta y salud cardiovascular. An Pediatr. 2011;74(3):192-206.

9. Ministério da Saúde. Guia alimentar para a população brasileira. 2006. [capturado 2012 nov 18]. Disponível em: http://dtr2001.saude.gov.br/editora/produtos/livros/pdf/05 1109_M.pdf.

10. Romanzini M, Reichert FF, Lopes AS, Petroski EL, Junior JCF. Prevalência de fatores de risco cardiovascular em adolescentes. Cad Saúde Pública. 2008;24(1):2573-81.

11. World Health Organization. WHO Child Growth Standards: body mass index-for-age: methods and development 
Geneva: WHO; 2007.

12. Pinheiro ABV, Lacerda AEM, Benzecry EH, Gomes MCS, Costa VM. Tabela para avaliação de consumo alimentar em medidas caseiras. São Paulo: Atheneu; 2008.

13. Institute of Medicine. Dietary Reference Intakes: applications in dietary assessment. Washington (DC): National Academy Press; 2001.

14. World Health Organization. Prevention of cardiovascular disease: guidelines for assessment and management of cardiovascular risk. Geneva: WHO; 2007.

15. World Health Organization. Global recommendations on physical activity for health. Geneva: WHO; 2010.

16. American Academy of Pediatrics. Children, adolescents, obesity and the media. Pediatrics. 2011;128(1):201-8.

17. Instituto Brasileiro de Geografia e Estatística. Pesquisa de Orçamentos Familiares 2008-2009: antropometria e estado nutricional de crianças, adolescentes e adultos no Brasil. Rio de Janeiro: IBGE; 2010.

18. Organização Pan-americana da Saúde. Estratégia e plano de ação regional para um enfoque integrado à prevenção e controle das doenças crônicas. Geneva: WHO; 2007.

19. Instituto Brasileiro de Geografia e Estatística. Pesquisa de Orçamentos Familiares 2008-2009: análise do consumo alimentar pessoal no Brasil. Rio de Janeiro: IBGE; 2011.

20. Pedersen TP, Meilstrup C, Holstein BE, Rasmussen M. Fruit and vegetable intake is associated with frequency of breakfast, lunch and evening meal: cross-sectional study of 11,13 , and 15 year olds. Int $\mathrm{J}$ Behav Nutr Phys Act. 2012; 9(9):1-10.

21. Vítolo MR. Hábitos alimentares na adolescência. In: Vítolo MR. Nutrição da gestação ao envelhecimento. Rio de Janeiro: Rubio; 2008. p.291-8.

\section{Endereço para correspondência:}

Marina Pioltine

Rua Barão de São Gabriel n 389

São Paulo/SP - CEP 05085-060

Telefone: +55 11999543343

E-mail: marinapioltine@usp.br 